\title{
Short-Term Electricity Price Forecasting Using a Combination of Neural Networks and Fuzzy Inference
}

\author{
Evans Nyasha Chogumaira, Takashi Hiyama \\ Department of Computer Science and Electrical Engineering, Kumamoto University, Kumamoto, Japan \\ E-mail:evans@st.cs.kumamoto-u.ac.jp \\ Received October 28, 201; revised November 3, 2010; accepted November 4, 2010
}

\begin{abstract}
This paper presents an artificial neural network, ANN, based approach for estimating short-term wholesale electricity prices using past price and demand data. The objective is to utilize the piecewise continuous nature of electricity prices on the time domain by clustering the input data into time ranges where the variation trends are maintained. Due to the imprecise nature of cluster boundaries a fuzzy inference technique is employed to handle data that lies at the intersections. As a necessary step in forecasting prices the anticipated electricity demand at the target time is estimated first using a separate ANN. The Australian New-South Wales electricity market data was used to test the system. The developed system shows considerable improvement in performance compared with approaches that regard price data as a single continuous time series, achieving MAPE of less than $2 \%$ for hours with steady prices and $8 \%$ for the clusters covering time periods with price spikes.
\end{abstract}

Keywords: Electricity Price Forecasting, Short-Term Load Forecasting, Electricity Markets, Artificial Neural Networks, Fuzzy Logic

\section{Introduction}

With deregulation in many electricity markets around the world, knowledge of possible future values of demand and the corresponding price has become more significant to the different entities on the market - generators and electricity traders for determining bidding strategies, and system operators for administration of the market [1].

Generally commodity prices are compelled by supply and demand balance. In electricity markets the traded 'commodity' cannot be stockpiled economically, the constraints are defined by the system total capacity to satisfy demand at any given time [2]. This therefore causes electricity prices to have a high probability of volatility, which masks observable trends necessary for forecasting future values, especially in the short term.

Short-term forecasts cover the period from a few minutes to about one week ahead. These are useful for dispatch and short-term or spot trading. Short term trading is meant to service the short-term variations in load and the actual prices are only known after matching of bids and offers by the market operator [1]. This presents a challenge in that to place effective bids; the traders need to have an idea of the future values of the demand and its corresponding price.

Different models have been employed in power systems for achieving forecasting accuracy and these include: regression, statistical and state space methods [3-6]. Artificial intelligence based approaches have been explored based on expert systems, evolutionary programming, fuzzy systems, artificial neural networks and various combinations of these. The widely used approach in previous works has been based on developing a mathematical model of the power system and then to perform simulations to determine required values [7-9]. The main challenge with this has been to make accurate non-linear mathematical models. Also, complete system data is not always readily available and the great computational effort required [2].

To estimate future values of any parameter one needs to have some information on factors that influence that parameter, or trends that describe the parameter of interest. A number of parameters have been analyzed to determine their usefulness as inputs data for short term estimation. These include weather variables, past demand and price data. Weather variables such as temperature used in [10] are either averaged information of forecasted values, there is always a chance that the uncertainty could 
affect output of price and load forecasting. As for past price, data analysis shows that trends are not maintained consistently for all hours of a day, or even for different week days.

Presented in this paper is an approach utilizing artificial neural networks (ANN) to extract trends from past data of the same parameters and use that to predict possible future values. A data clustering approach is adopted that groups the data into sets with cut off points that vary from one day to the other. This creates zones of intersection, and to handle these points fuzzy logic is used.

In the wholesale electricity markets different types of prices exist, but the most basic pricing concept is the market clearing price (MCP). When there is transmission congestion in the system then other prices concepts such as the location marginal price (LMP) and the zonal market clearing price (ZMCP) are used. This paper focuses on MCP which is not dependent on internal transmission constraints.

\section{Selecting Input Data}

A variety of parameters have generally been used as input to price and demand forecasting by different researchers, from weather variables, socio-economic indices, time indicators to past trends of the demand and its corresponding price. One approach would be to adopt techniques used in long term forecasts, which uses most of the factors stated above, but the disadvantage of these is that useful information about changes during price spikes is discarded as outliers [11-13].

Statistical analysis was used to find the dominant factors that decide the present electricity cost. An overview of price and demand variation for a week, shown in Figure 1 below, shows that the price variation is a complex trend that requires detailed analysis to establish the influencing factors. The figure also shows that demand varies with some readily observable repetitive pattern yet in the same period the price shows spikes of varying magnitude and duration.

Weather variables were also considered; the parameters of interest are temperature and cloud cover. From Figure 2 we can see that temperature and cloud cover are similar in terms of information they provide. Observable correlation is over a long period of time and in the short term weather variables cannot be relied on as input to load forecasting.

Socio-economic variables are performance indicators like gross domestic product, which are complex figures incorporating estimates and averages making them not the best inputs for further estimation. Significant social activities are strongly linked to the calendar days and hours of the day such that simply including an element of time

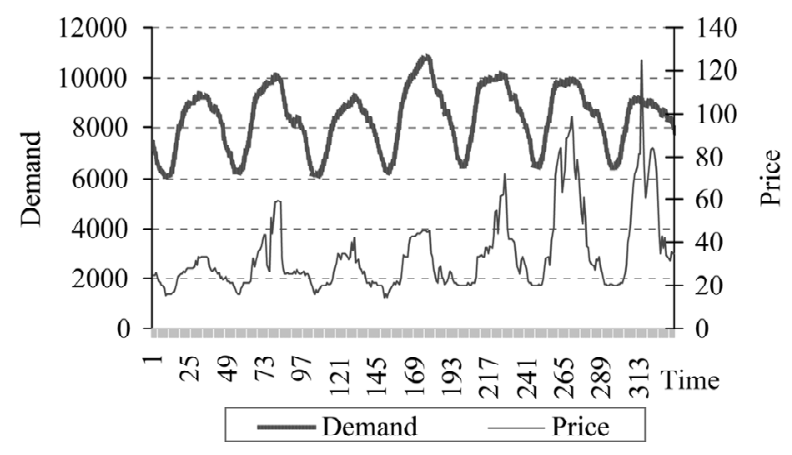

Figure 1. Price and demand variations for a week.

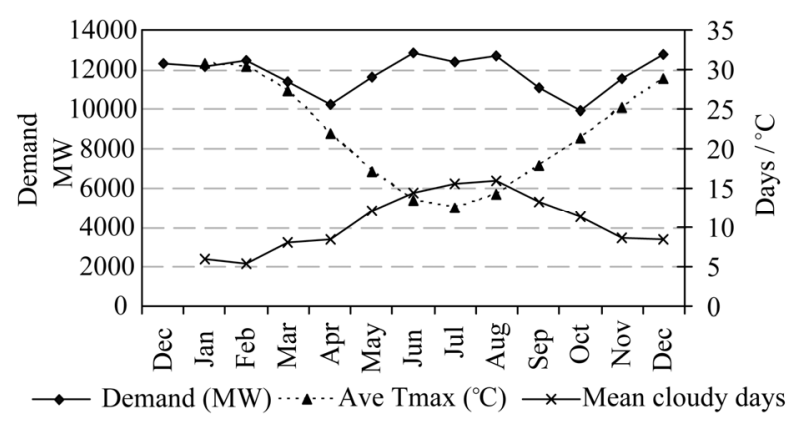

Figure 2. Weather variables and electricity demand.

in the input obviates them.

Past data has been selected for this approach other as factors are either estimates in themselves or data averaged over a long time making them not ideal for shortterm application. For past price data a similarity index was calculated for past demand and past price values, defined as follows:

$$
\mathbf{J}_{\mathrm{MT}}=\left\{\sum\left[\mathbf{P}_{\mathrm{DM}(\mathrm{k})}-\mathbf{P}_{\mathrm{DT}(\mathrm{k})}\right]^{2}\right\}^{1 / 2}
$$

Where,

$\mathrm{P}_{\mathrm{DM}}(\mathrm{k})$ - parameter value on the target day for hour $\mathrm{k}$

$\mathrm{P}_{\mathrm{DT}}(\mathrm{k})$ - parameter value from past data for hour $\mathrm{k}$

$\mathrm{k}$ - sampling interval

The smaller the figure is the higher is the similarity in the compared days. The similarity indices show a very significant difference from one day to the next, so to avoid masking trends by grouping data from different days; each day of the week is treated individually. National holidays are treated as Sundays. Further correlation analysis was done through scatter plots shown in Figures 3-5.

From the scatter plots we can easily see that the price variation between different days can be described by more than one trend, and therefore cannot be easily approximated by a single function. As for the demand the relationship between one day's data set and the next is nearly linear.

In Figure 5 the comparison between price and demand 


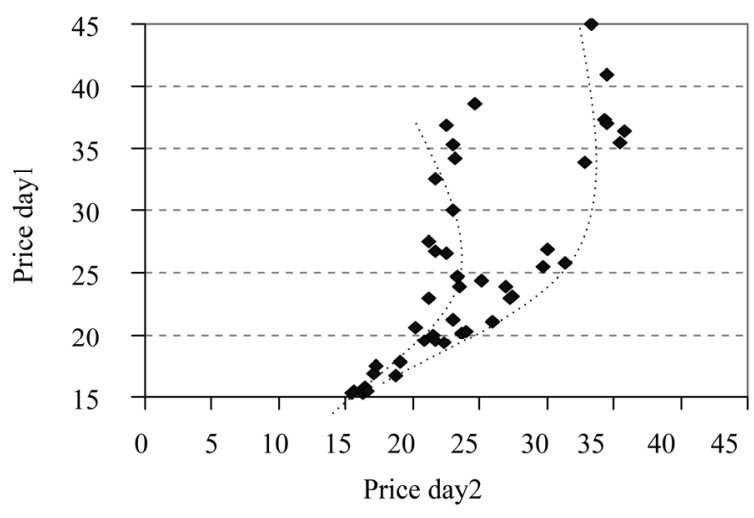

Figure 3. Correlation between different days for price.

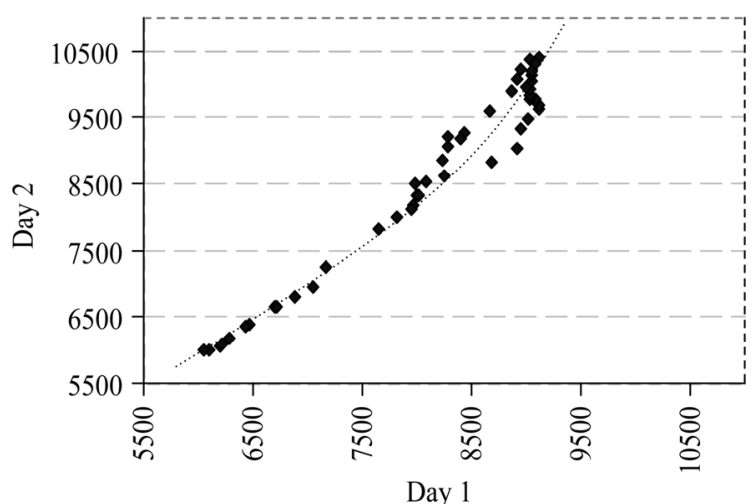

Figure 4. Correlation between different days for demand.

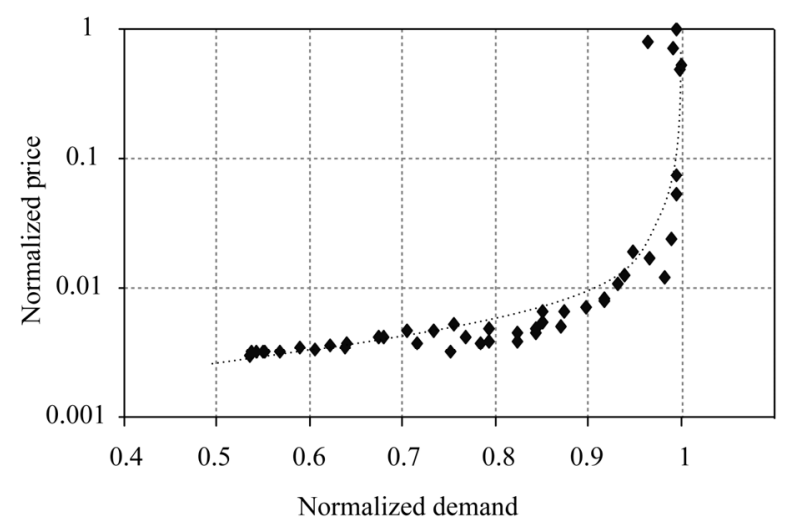

Figure 5. Price against demand.

shows an almost linear relationship up to some point at which there is a sudden rise in price. This shows that there is a significant change in the dominant factors that determine the price at some level of demand, suggesting that price can be regarded as having multiple trends for which calculations can be done separately.

Published market data shows the power served, which is almost always the power demanded. However, information about the supplying generators is not readily available publicly, yet this has more influence on the price than the magnitude of demand. Normally expensive generators are dispatched last, so at high demand periods the price demand ratio tends to be much higher, as can be seen in Figure 5.

\section{System Configuration and Training}

\subsection{Artificial Neural Networks}

An artificial neural network (ANN) is a model that emulates the functional architecture of the human brain. In this research a multi-layer perceptron ANN is adopted. This ANN consists of: an input layer, hidden layers and an output layer as shown in Figure 6. Except for the input layer, each neuron receives a signal that is a linearly weighted sum of the outputs from all the neurons in the preceding layer.

Activation of neuron $j$ is then defined as

$$
U_{j}^{(k)}=f_{i}\left\{\sum_{i} w_{j i} i_{i}^{(\mathrm{k}-1)}\right\}
$$

Where, if neuron $j$ is a hidden neuron, $f_{i}(x)=[1+\exp (-x)]^{-1}$. Otherwise, $f_{i}(x)=x$. $i$ covers all the neurons in the in the layer $(k-1)$.

Note, the activation of the $j$ th neuron in the $k$ th layer, $u_{j}^{(k)}$ in (1), is only a function of the activations of the neurons in the $(k-1)$ th layer and the weights which connect the $j$ th neuron in the $k$ th layer with the neurons in the $(k-1)$ th layer. The non-linearity $f_{j}(x)$ can be any monotonic function differentiable in the $x$ domain [14].

\subsection{Fuzzy Inference Systems}

Fuzzy inference is an implementation of fuzzy logic in which linguistic like rules map the input onto output space without strict specification of the input $[3,4]$.

Fuzzy Logic: if $\mathrm{X}$ is a universe of discourse with elements denoted by $x$, then the fuzzy set $\mathrm{A}$ in $\mathrm{X}$ is defined as a set of ordered pairs, $\mathrm{A}=\left\{x, \mu_{A}(x) \mid x \in X\right\} . \mu_{A}(x)$ is called the membership function of $x$ in A. Figure 7 shows

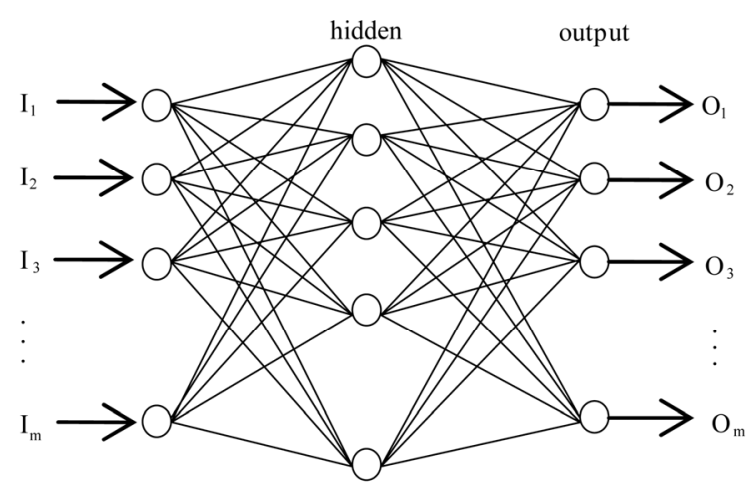

Figure 6. Multi layer perceptron type ANN. 


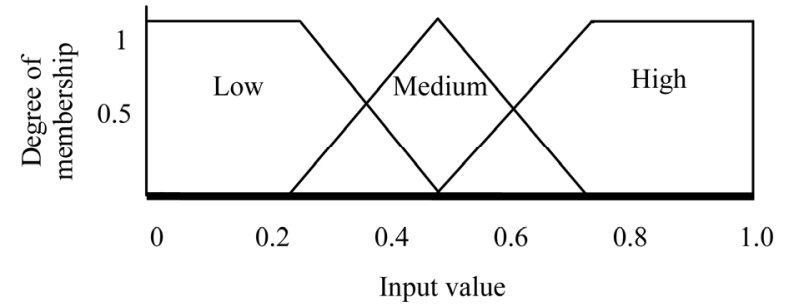

Figure 7. Triangular membership function.

the triangular membership function used for three different fuzzy sets; Low, Medium and High.

Fuzzy inference systems (FIS) use fuzzy rules (IF Then) and fuzzy reasoning, an inference procedure that obtains conclusions from a set of fuzzy rules and known facts. Three conceptual components: rule base (fuzzy rules selection); database (membership functions) and reason mechanism inference procedure thus form the basic structure of a fuzzy inference system.

\subsection{System Configuration}

A number of configurations based on the proposed approach were setup. A system with cascaded processing elements was setup as shown in Figure 8 below. Included are data conditioning and classification blocks as part of preprocessing and post-processing for the price estimation path.

\subsection{Short-Term Demand Estimation}

Short-term load forecasting (STLF) is implemented through a single stage ANN calculation process. Two input configurations are taken for comparison. They both use past demand data as input; supplied concurrently or sequentially. Sequential inputs - input vectors occur in a specific time order. Concurrent inputs - input ordering not important as the inputs do not interact with each other. A separate ANN was trained for each day of the week.

\subsection{Short-Term Price Estimation}

For short-term price estimation the estimation system was setup in two configurations, one with data clustering and the other without, for comparison. The preprocessing block in the first case (referred to as method-1) has logic for data clustering and normalizing, i.e., mapping the data onto the [0 to 1$]$ domain. The clusters are defined as follows:

1) normal variations

2) sharply rising prices

3) sharply falling prices

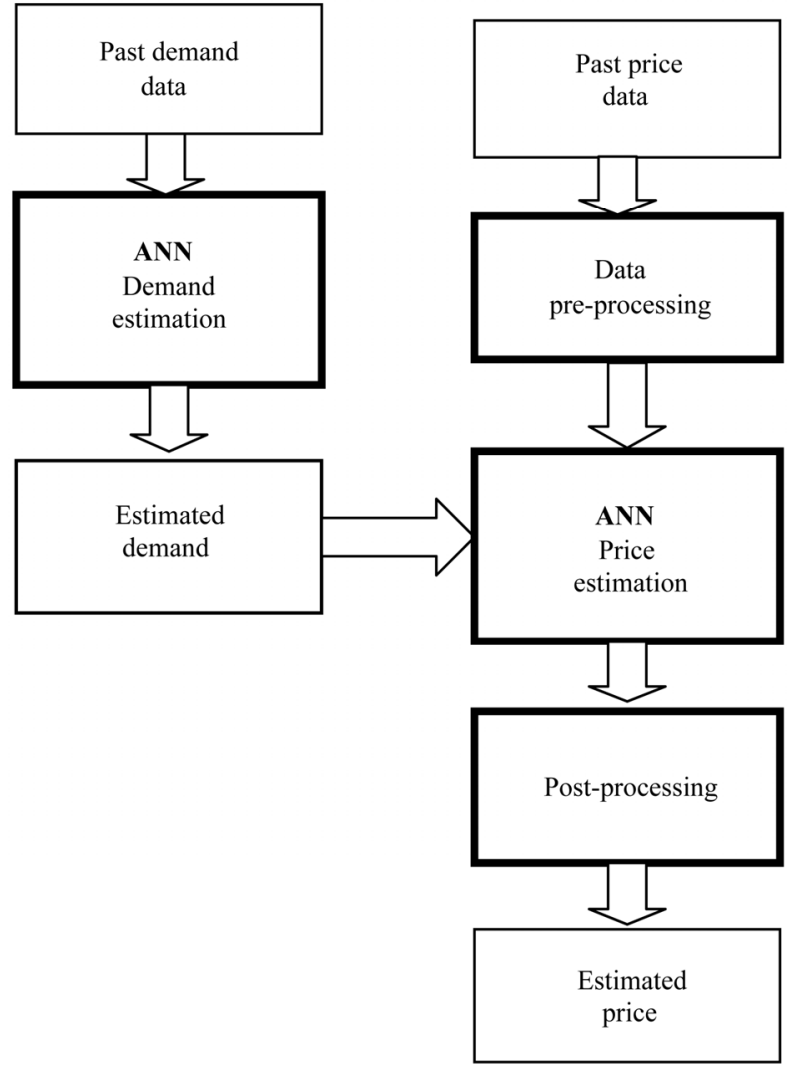

Figure 8. System configuration.

Figure 9 shows the moving average and the derivative taken for a typical weekday.

$$
\mathrm{P}(t)=\left\{\begin{array}{l}
\mathrm{A} ; \mathrm{AD} \leq * \mathrm{MA} \\
\mathrm{B} ; \mathrm{AD} \geq 2 * \mathrm{MA} \&|\mathrm{FD}|>0 \\
\mathrm{C} ; \mathrm{AD} \geq 2 * \mathrm{MA} \&|\mathrm{FD}| \leq 0
\end{array}\right.
$$

Where, AD - Actual price data

MA - moving average of price data

FD - first derivative of the price data

$$
\mathrm{FD}=\frac{\Delta P}{\Delta t}=\frac{P_{n+1}-P_{n}}{\Delta t}
$$

In the second configuration, method-2, instead of clustering, preprocessing is done by logarithmic conditioning as in (4).

$$
\mathrm{P}_{\text {in }}(t)= \begin{cases}\mathrm{P}(t) ; & \mathrm{P}(t) \leq * \mathrm{UL} \\ \mathrm{UL}+\mathrm{P}(t) * \log [\mathrm{P}(t) / \mathrm{UL}] ; \mathrm{P}(t) \geq \mathrm{UL}\end{cases}
$$

Post processing block for method-1 contains the fuzzy inferencing logic for the data cluster intersections. Choice of membership function is influenced by need for simplicity, convenience, speed and efficiency. The triangular membership function chosen for these reasons.

For the second configuration post processing block has 


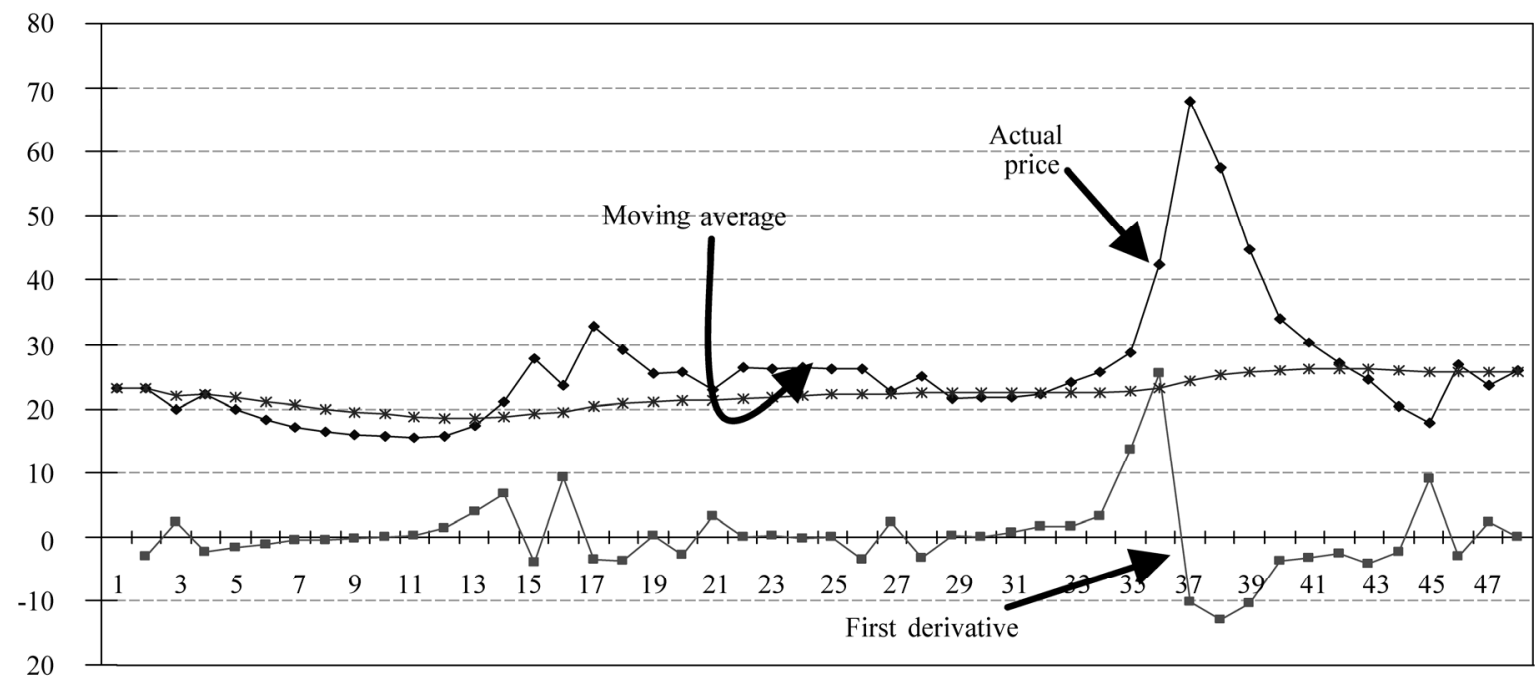

Figure 9. Actual price, first derivative and moving average.

the logic for data recovery, shown in (5):

$$
\mathrm{P}_{\text {out }}(t)= \begin{cases}\mathrm{P}_{\text {out }}(t) ; & \mathrm{P}_{\text {out }}(t) \leq \mathrm{UL} \\ \mathrm{UL} * \exp [(\mathrm{P}(t)-\mathrm{UL}) / \mathrm{UL}] ; & \mathrm{P}_{\text {out }}(t) \geq \mathrm{UL}\end{cases}
$$

\subsection{Training}

The proposed system was setup in the various configurations in a Matlab environment. The ANN blocks were initialized with randomly generated numbers as weights then trained on past data. Back propagation with gradient descent method was used for updating the weights. The training performance measure used is the mean square error, MSE:

$$
\operatorname{MSE}=\frac{1}{N} \sum\left(\mathrm{V}_{\mathrm{a}}(i)-\mathrm{V}_{\mathrm{f}}(i)\right)^{2}
$$

The optimum number of training vectors/patterns required to classify test examples with an error limit of $\delta$ approximately equal to the number of weights in the network multiplied by the inverse of the error limit [15]. This was used as a guideline in preparing the input data for training. The training performance for the three data clusters A, B and C is shown in Figure 10.

Triangular membership function was used for the fuzzy inference (FIS) and the rules incrementally developed in the tuning process. The inputs supplied to the FIS block are the estimated price for the intersection region and the estimated demand for the target hours.

Tuning the fuzzy inference system was done by changing the rule antecedents or conclusions, changing the centers of the input and/or output membership functions based on the error between the target output and calculated value. No predetermined method was used for the tuning, but trial and error approach seeking to modify only the components pulling the output in the direction of the error.

\section{Simulation and Results}

After training the different network configurations were tested with the data sets not used for training to measure the performance. The platform for the simulation was a Windows desktop computer with 2 GB of RAM. The post training computer executions during simulations were almost instantaneous, although speed was not the most critical factor in this study as the system is for offline not real-time forecasting. The following sections show the test results and analysis. The performance measure used is the mean average percentage error, MAPE, defined as:

$$
\mathrm{MAPE}=\frac{1}{N} \sum_{i}\left|\mathrm{~V}_{\mathrm{a}}(i)-\mathrm{V}_{\mathrm{f}}(i)\right|
$$

Table 1 shows the estimation performance for demand

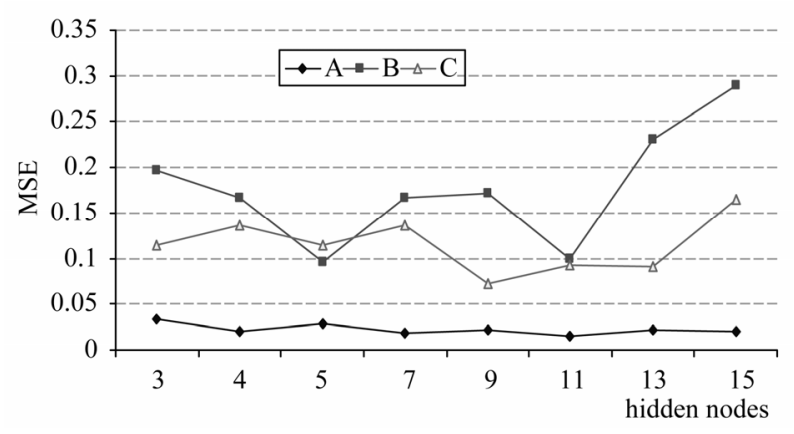

Figure 10. Ann training for price estimation. 
Table 1. Demand forecasting results.

\begin{tabular}{lcc}
\hline Input vector elements & Network size & MAPE \\
\hline 2 weeks past demand & $96-72-48$ & 2.3 \\
3 weeks past demand & $144-96-48$ & 3.5 \\
4 weeks past demand & $192-144-48$ & 10.3 \\
& $6-3-1$ & 2.5 \\
Past demand values for 3 & $6-4-1$ & 2.02 \\
immediate hours, & $6-5-1$ & 2.43 \\
$\begin{array}{l}\text { Demand values for the same } \\
\text { hour as target hour from 3 }\end{array}$ & $6-7-1$ & 2.25 \\
previous weeks & $6-8-1$ & 2.21 \\
& $6-10-1$ & 6.09 \\
\hline
\end{tabular}

forecasting in the two configuration variations. Results shown in Table 1 above show that demand estimation in the short-term can be achieved with good accuracy through a well trained ANN. The difference in the two variations is mostly due to the network size; the higher dimension networks require a lot of training data before it can be able to generalize.

Figures 11 and 12 show the results for the estimation plotted against the target for demand. The main reasons for the estimation performance shown for demand with a single stage ANN are: the consistency in trends for the demand data and the grouping/clustering of demand data into similar weekdays.

Results for price estimation using method- 1 are shown in Tables 2-4 for each data cluster. The input vectors comprise of: estimated demand at target hour and values for the same hour as target, from 4 previous weeks for the 5-input networks. For the 9-inputs networks price values for 4 immediate past hours are also added.

Results shown in the tables also illustrate the effect of changing the size of the input vector on performance. Forecasting accuracy for cluster A is significantly better than the other clusters because it represents hours of steady variation in price. Figures 13-15 illustrate the level of coincidence between the forecasted values and target for each cluster. Figure $\mathbf{1 6}$ shows the forecasted values for hours falling in the intersection of cluster $A$ and cluster B.

Results for forecasting using method-2 are shown in Table 5. Increasing input vector size, by increasing the number of weeks of past data initially provides a wider variety of data to the ANN, but beyond a certain network size the ANN loses ability to generalize. Figure $\mathbf{1 7}$ also shows output obtained with this method.

\section{Conclusions}

Electricity price in the short-term shows high volatility

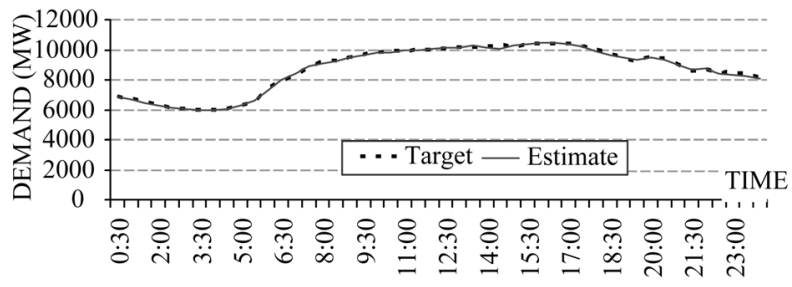

Figure 11. Demand estimation network size [6-4-1] with sigmoid transfer function.

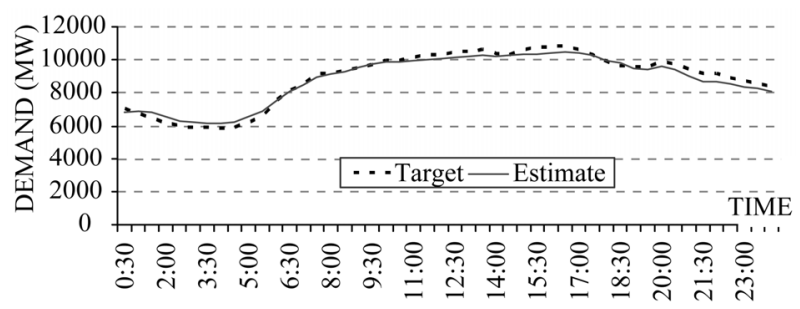

Figure 12. Demand estimation linear network [6-4-1].

Table 2. Forecasting results for cluster $\mathrm{A}$.

\begin{tabular}{cccc}
\hline Network size & MAPE & Network size & MAPE \\
\hline $5-5-1$ & 1.065 & $9-5-1$ & 1.870 \\
$5-8-1$ & $\mathbf{0 . 5 9 8}$ & $9-8-1$ & 0.914 \\
$5-11-1$ & 1.312 & $9-11-1$ & $\mathbf{0 . 7 0 4}$ \\
$5-13-1$ & 0.944 & $9-13-1$ & 1.343 \\
$5-14-1$ & 0.813 & $9-14-1$ & 1.451 \\
\hline
\end{tabular}

Table 3. Forecasting results for cluster $B$.

\begin{tabular}{cccc}
\hline Network size & MAPE & Network size & MAPE \\
\hline $5-5-1$ & 3.0 & $9-5-1$ & 9.7 \\
$5-7-1$ & 6.3 & $9-7-1$ & 16.7 \\
$5-9-1$ & 4.6 & $9-9-1$ & 17.2 \\
$5-11-1$ & 3.6 & $9-11-1$ & 9.9 \\
$5-13-1$ & 6.6 & $9-13-1$ & 23.1 \\
\hline
\end{tabular}

Table 4. Forecasting results for cluster $\mathrm{C}$.

\begin{tabular}{cccc}
\hline Network size & MAPE & Network size & MAPE \\
\hline $5-7-1$ & 3.3 & $9-7-1$ & 13.7 \\
$5-9-1$ & $\mathbf{2 . 0}$ & $9-9-1$ & $\mathbf{7 . 3}$ \\
$5-11-1$ & 2.4 & $9-11-1$ & 9.2 \\
$5-13-1$ & 1.9 & $9-13-1$ & 9.1 \\
$5-15-1$ & 2.6 & $9-15-1$ & 16.5 \\
\hline
\end{tabular}




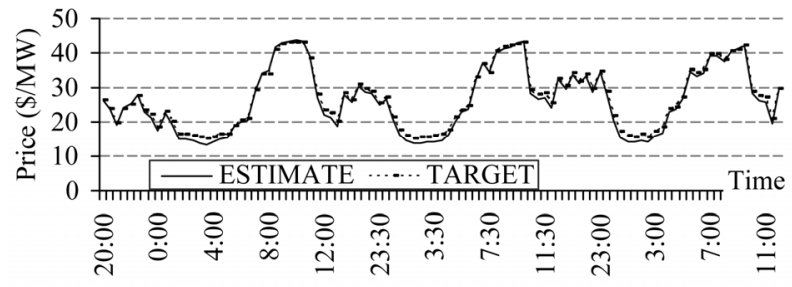

Figure 13. Price estimation method-1: cluster A.

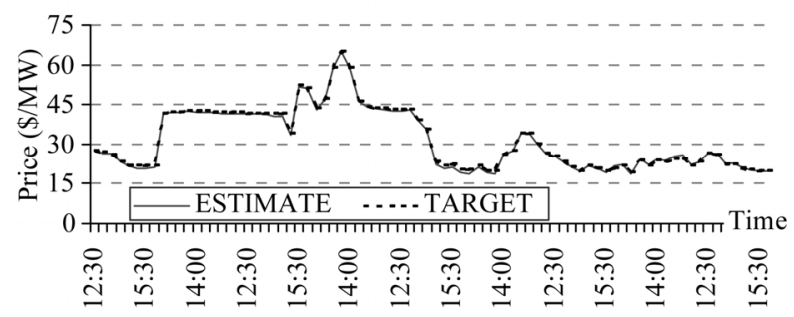

Figure 14. Price estimation method-1: cluster $B$.

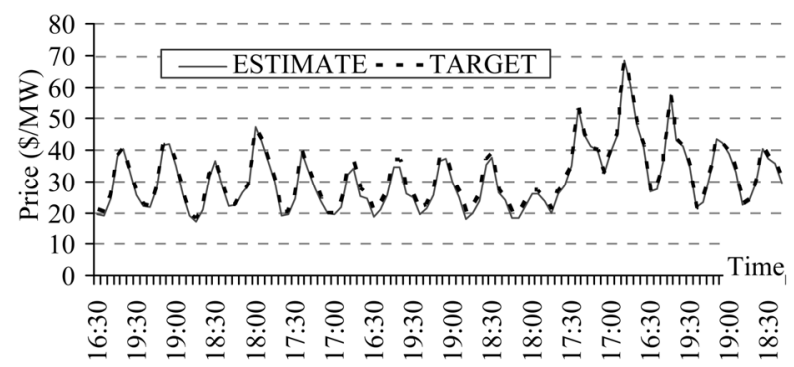

Figure 15. Price estimation method-1: cluster $C$.

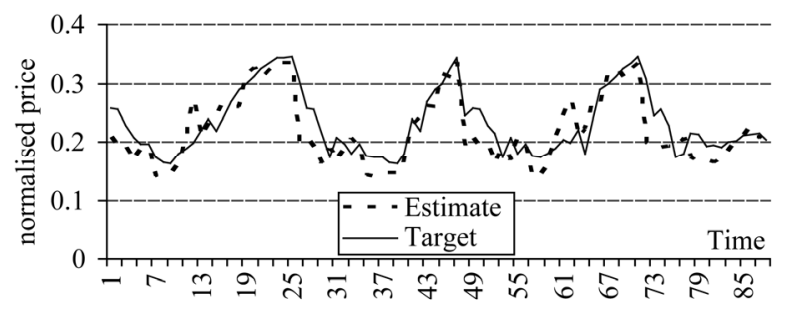

Figure 16. Price estimation method-1: intersection AB.

making it difficult to predict its future value, but with the proposed approach significant improvement in the forecasting performance has been observed. One key element in the processing is selection of input data and careful preprocessing. Data clustering into ranges in which observable trends are maintained allows for the extraction of such trends using separate ANN trained for each specific cluster. Data samples that cannot fit into the strictly defined clusters form intersection zones that, in addition to ANN, are processed using fuzzy inference.

Electricity demand shows smoothly varying trends such that a single stage well tuned ANN blocked managed to achieve good accuracy. Of the two configuration
Table 5. Price forecasting results using method-2.

\begin{tabular}{cccc}
\hline Case & Input vector elements & Network size & MAPE \\
\hline 1 & $\begin{array}{c}\text { Forecasted demand } \\
\text { 1 week past price }\end{array}$ & $96-72-48$ & 18 \\
2 & $\begin{array}{c}\text { Forecasted demand } \\
\text { 2 week past price }\end{array}$ & $144-96-48$ & 21 \\
3 & $\begin{array}{c}\text { Forecasted demand } \\
\text { 3 week past price }\end{array}$ & $192-144-48$ & $\mathbf{8}$ \\
4 & $\begin{array}{c}\text { Forecasted demand } \\
\text { 4 week past price }\end{array}$ & $240-192-48$ & 17 \\
\hline
\end{tabular}

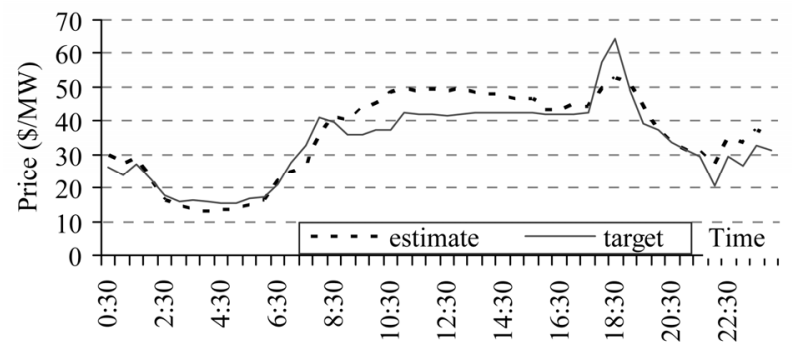

Figure 17. Price estimation method-2.

options tested comparable performance was obtained. However, the second configuration that uses large input vectors comprising of complete days data requires more computational effort, so the first configuration is considered optimum.

For electricity price estimation data clustering gives a comparatively much higher accuracy for the normal price region and a significantly better result for the times characterized with spikes. It is therefore concluded that the way to approach electricity price forecasting while maintaining all the data is to use a method that employs data clustering even within a day's data set.

\section{References}

[1] M. Shahidehpour, H. Yamin and Z. Li, "Market Operations in Electric Power Systems," John Wiley \& Sons, Chichester, 2002. doi:10.1002/047122412X

[2] A. K. Topalli, I. Erkmen and I. Topalli, "Intelligent Short-term Load Forecasting in Turkey," Electrical Power and Energy Systems, Vol. 28, 2006, pp. 437-447. doi: 10.1016/j.ijepes.2006.02.004

[3] R. C. Garcia, et al., "GARCH Forecasting Model to Predict Day-ahead Electricity Prices," IEEE Transactions on Power Systems, Vol. 20, No. 2, May 2005, pp. 867-874. doi:10.1109/TPWRS.2005.846044

[4] M. Stevenson, "Filtering and Forecasting Spot Electricity Prices in the Increasingly Deregulated Australian Electricity Market,” Quantitative Finance Research Centre, University of Technology, Sydney, 2001.

[5] N. Hubele, et al., "Identification of Seasonal Short-term 
Load Forecasting Models Using Statistical Decision Functions,” IEEE Transactions on Power Systems, Vol. 5, No. 1, 1990, pp. 40-5. doi:10.1109/59.49084

[6] M. El-Hawary, et al, "Short-Term Power System Load Forecasting Using the Iteratively Reweighted Least Squares Algorithm,” Electrical Power Systems Research, Vol. 19, 1990, pp. 11-22. doi:10.1016/0378-7796(90)900 03-L

[7] V. S. Kodogiannis and E. M. Anagnostakis, “A Study of Advanced Learning Algorithms for Short-term Load Forecasting," Engineering Applications of Artificial Intelligence, Vol. 12, 1999, pp. 159-173. doi:10.1016/S09521976(98)00064-5

[8] G.-C. Liao and T.-P. Tsao, “Application of Fuzzy Neural Networks and Artificial Intelligence for Short-term load Forecasting,” Electrical Power Systems Research, Vol. 70, 2004, pp. 237-244. doi:10.1016/j.epsr. 2003.12.012

[9] H. Yamin, M. Shahidehpour and Z. Li, “Adaptive short-term Price Forecasting using artificial Neural Networks in the Restructured Power Markets," Electrical Power and Energy Systems, Vol. 26, 2004, pp. 571-581. doi:10.1016/j.ijepes.2004.04.005

[10] P. Mandal, T. Senjyu, N. Urasaki and T. Funabashi, “A
Neural Network Based Several-Hour-Ahead Electric Load Forecasting using Similar Days Approach,” Electrical Power and Energy Systems, Vol. 28, 2006, pp. 367-373. doi:10.1016/j.ijepes.2005.12.007

[11] S. Rahman and R. Bhatnager, "An Expert System based Algorithm for Short Term Load Forecast," IEEE Transactions on Power Systems, Vol. 3, No. 2, 1988, pp. 392399. doi:10.1109/59.192889

[12] Q. Lu, et al., “An Adaptive Nonlinear Predictor with Orthogonal Escalator Structure for Short-term Load Forecasting," IEEE Transactions on Power Systems, Vol. 4, No. 1, 1989, pp. 158-164. doi:10.1109/59.32473

[13] I. Moghram and S. Rahman, "Analysis and Evaluation of Five Short-Term Load Forecasting Techniques," IEEE Transactions on Power Systems, Vol. 4, No. 4, 1989, pp. 1484-1491. doi:10.1109/59.41700

[14] M. Ikeda and T. Hiyama, “ANN Based Designing and Cost Determination System for Induction Motor," IEEE Proceeding Electrical Power Application, Vol. 152, No. 6, 2005, pp. 1595-1602. doi:10.1049/ip-epa:20050173

[15] E. Baum and D. Haussler, "What Net Size Gives Valid Generalization?” Neural Computation, Vol. 1, No. 1, 1989, pp. 151-160. doi:10.1162/neco.1989.1.1.151 